\title{
Natural killer cell immunosuppressive function requires CXCR3-dependent redistribution within lymphoid tissues
}

\author{
Ayad Ali, ${ }^{1,2,3}$ Laura M. Canaday, ${ }^{2,3}$ H. Alex Feldman, ${ }^{1,2,3}$ Hilal Cevik, ${ }^{3,4}$ Michael T. Moran, ${ }^{2,3}$ Sanjeeth Rajaram, ${ }^{3,5}$ Nora Lakes, ${ }^{1,2}$ \\ Jasmine A. Tuazon, ${ }^{3}$ Harsha Seelamneni, ${ }^{3}$ Durga Krishnamurthy, ${ }^{3}$ Eryn Blass, ${ }^{6}$ Dan H. Barouch, ${ }^{6,7}$ and Stephen N. Waggoner ${ }^{1,2,3,4,8}$ \\ ${ }^{1}$ Medical Scientist Training Program and ${ }^{2}$ Immunology Graduate Training Program, University of Cincinnati College of Medicine, Cincinnati, Ohio, USA. ${ }^{3}$ Center for Autoimmune Genomics and Etiology, \\ Cincinnati Children's Hospital Medical Center, Cincinnati, Ohio, USA. ${ }^{4}$ Molecular and Developmental Biology Graduate Program and ${ }^{5}$ Medical Sciences Program, University of Cincinnati College of Medicine, \\ Cincinnati, Ohio, USA. ${ }^{6}$ Center for Virology and Vaccine Research, Beth Israel Deaconess Medical Center, Harvard Medical School, Boston, Massachusetts, USA. ${ }^{7}$ Ragon Institute of MGH, MIT, and Harvard, \\ Cambridge, Massachusetts, USA. ${ }^{8}$ Department of Pediatrics, University of Cincinnati College of Medicine, Cincinnati, Ohio, USA.
}

NK cell suppression of T cells is a key determinant of viral pathogenesis and vaccine efficacy. This process involves perforindependent elimination of activated $\mathrm{CD4}^{+} \mathrm{T}$ cells during the first 3 days of infection. Although this mechanism requires cell-cell contact, NK cells and T cells typically reside in different compartments of lymphoid tissues at steady state. Here, we showed that NK cell suppression of T cells is associated with transient accumulation of NK cells within T cell-rich sites of the spleen during lymphocytic choriomeningitis virus infection. The chemokine receptor CXCR3 was required for this relocation and suppression of antiviral T cells. Accordingly, NK cell migration was mediated by type I IFN-dependent promotion of CXCR3 ligand expression. In contrast, adenoviral vectors that weakly induced type I IFN and did not stimulate NK cell inhibition of T cells also did not promote measurable redistribution of NK cells to T cell zones. Exogenous IFN rescued NK cell migration during adenoviral vector immunization. Thus, type I IFN and CXCR3 were critical for properly positioning NK cells to constrain antiviral T cell responses. Development of strategies to curtail migration of NK cells between lymphoid compartments may enhance vaccine-elicited immune responses.

\section{Introduction}

NK cells are innate lymphocytes with a critical role in immune defense against viruses in both mice and humans $(1,2)$. In addition to killing virus-infected cells, NK cells regulate antiviral T and $\mathrm{B}$ cell responses via perforin-dependent elimination of activated $\mathrm{CD}^{+} \mathrm{T}$ cells $(3,4)$. Lymphocytic choriomeningitis virus (LCMV) infection potently triggers immunoregulatory functions of NK cells, with significant consequences for viral clearance and immune pathology (3-5). Similar mechanisms constrain humoral immunity after immunization of mice $(6,7)$. NK cell suppression of $\mathrm{T}$ cell or antibody responses is also apparent in humans during infections with HIV or hepatitis B virus (HBV) and after administration of yellow fever or HBV vaccines (8-11). An improved understanding of the mechanisms of NK cell immunosuppressive activity is likely to facilitate development of interventions to enhance vaccine efficacy.

NK cell suppression of $\mathrm{T}$ cells has been reproducibly linked to perforin $(3-6,12)$, a key component of cell-contact-depen-

Related Commentary: https://doi.org/10.1172/JCI152054

Conflict of interest: The authors have declared that no conflict of interest exists. Copyright: (5) 2021, American Society for Clinical Investigation.

Submitted: December 14, 2020; Accepted: July 22, 2021; Published: September 15, 2021 Reference information: J Clin Invest. 2021;131(18):e146686.

https://doi.org/10.1172/JCl146686. dent granule-mediated killing (13). Thus, the immunoregulatory activity of NK cells likely involves physical liaisons with T cells in lymphoid tissues. Yet in the absence of inflammation, NK cells are infrequently present at $\mathrm{T}$ cell-rich sites, including lymph nodes and the white pulp (WP) of the spleen (14-17). Moreover, NK cells present in human lymph nodes typically express little perforin (18). However, infections with murine cytomegalovirus (MCMV) or LCMV trigger NK cell accumulation in the WP of spleen (1416), while infections of humans and nonhuman primates with HIV or SIV result in localization of NK cells into T cell and/or B cell-rich follicles (19). In this study, we establish that positioning of NK cells at T cell-rich sites is essential for NK cell suppression of antiviral $\mathrm{T}$ cells.

\section{Results and Discussion}

Transient positioning of $N K$ cells in $T$ cell zones during infection. NK cells eliminate a fraction of virus-specific $\mathrm{CD} 4^{+} \mathrm{T}$ cells via a perforin- and contact-dependent manner in the spleen during the initial 3 days of LCMV infection in mice $(3,4)$. Yet at baseline, NKp $46^{+} \mathrm{NK}$ cells predominately populate the red pulp $(\mathrm{RP})$ regions of the mouse spleen, with few NK cells detectable in the T cell-rich WP (Figure $1 \mathrm{~A}$ and Supplemental Figure 1; supplemental material available online with this article; https://doi.org/10.1172/JCI146686DS1). Consistent with previous reports $(14,16)$, infection with LCMV triggered increased proportions (Figure 1, B and C) and numbers (Figure 1D) of NKp46+ NK cells in the WP (Figure 1, B and D) and 
A

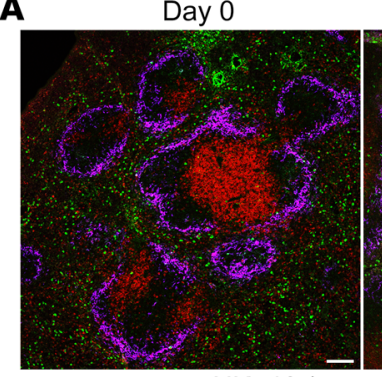

NKp46 (green)

B

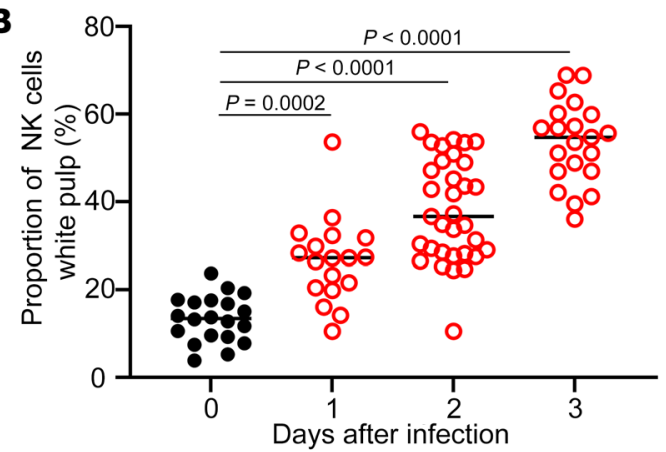

D

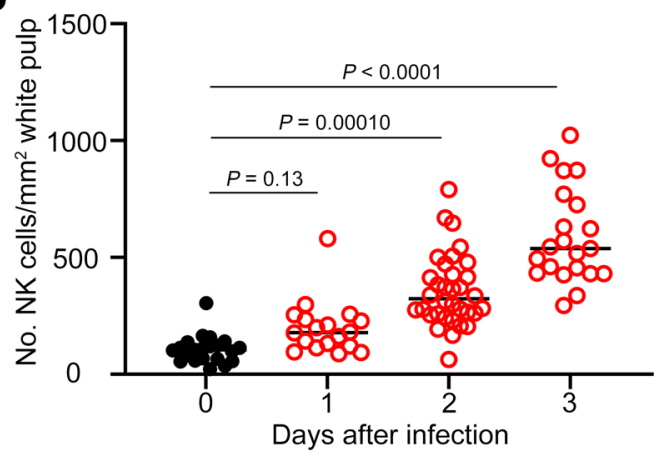

Day 1

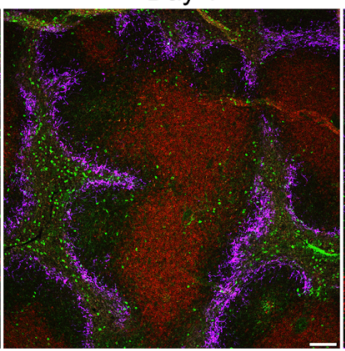

CD3 (red)

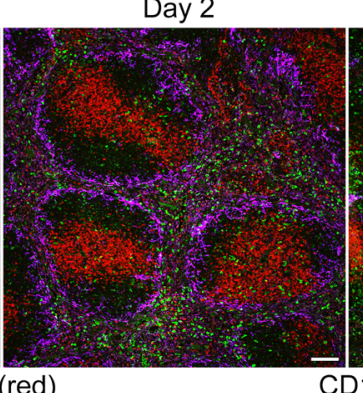

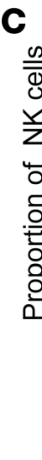

E
CD169 (purple)

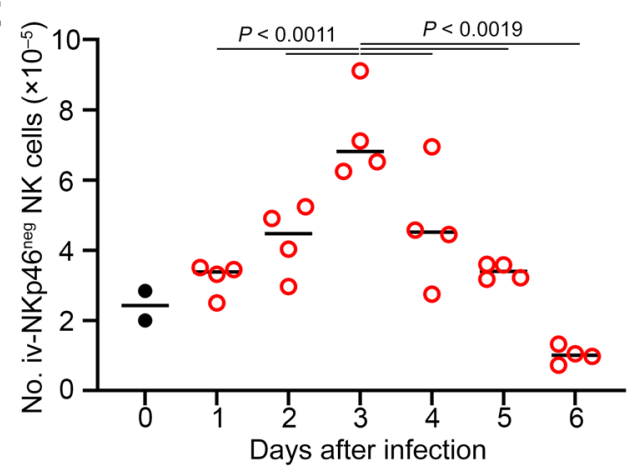

F

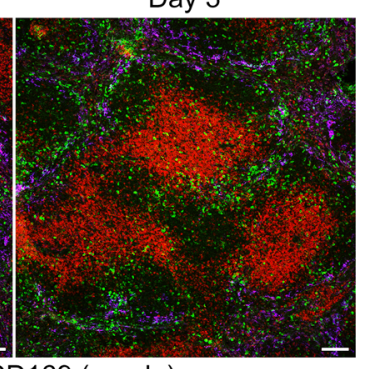

$P<0.0001$

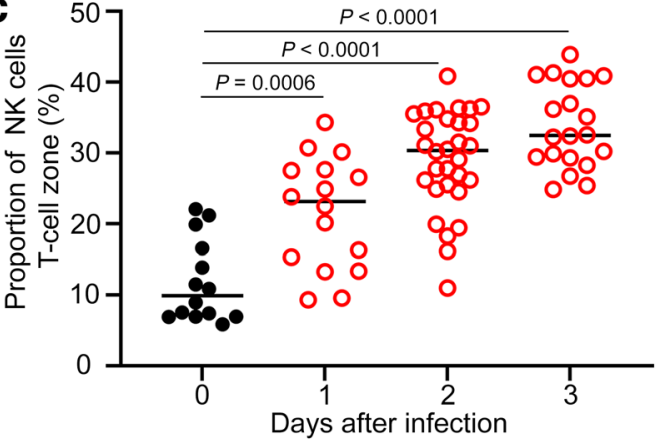

(1) \\ 36.}

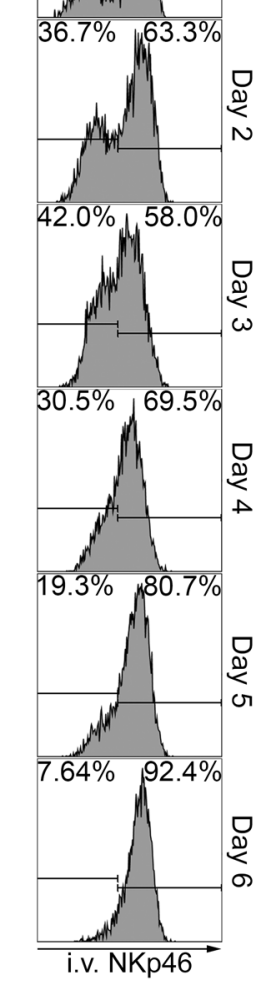

Figure 1. Transient positioning of NK cells in T cell zones during infection. (A-F) C57BL/6 mice ( $n=3-4 / g r o u p)$ were infected with the Armstrong strain of LCMV. Proportion (B and C) and number (D) of NKp46+ NK cells (green) enumerated within (B and $\mathbf{D})$ CD169 macrophage-bound (purple) WP or in (C) CD3 ${ }^{+}$T cell zones (red) is plotted (5-12 follicles/mouse). (E and F) Mice were intravenously injected at indicated times points with anti-NKp46 antibody 3 minutes prior to euthanasia to label splenic NK cells (CD3 ${ }^{\text {neg TCR }} \beta^{\text {neg } C D 8} \alpha^{\text {neg }}$ CD49b+NK1.1+ex-vivoNKp46+) in RP (iv-NKp46+) or WP (iv-NKp46 neg) regions. (E) Mean number of iv-NKp46 neg NK cells is graphed (4 mice/time point). (F) Representative histograms of iv-NKp46 staining of gated NK cells. Data are representative of 2 independent experiments, with statistical differences determined by 1-way ANOVA. Scale bars: $100 \mu \mathrm{m}$.

$\mathrm{T}$ cell zones (Figure 1C). This relocalization was apparent at 24 hours and continued to increase until roughly $60 \%$ of splenic NK cells localized within the WP by day 3 of infection (Figure 1, A-D).

To quantify NK cell localization, we took advantage of differences in vascularity between spleen RP and WP that can be detected using a modification of established intravascular staining methods (20). We intravenously injected an APC-labeled anti-NKp46 antibody and euthanized mice after 3 minutes. Ex vivo staining with anti-NKp46 ubiquitously labeled splenic RP NK cells in both infected and uninfected mice (Supplemental Figure 2, A and B). Consistent with restricted labeling of NK cells in poorly vascularized sites by intravenous anti-NKp46 antibody (iv-NKp46), NK cells in the WP were shielded from intravascular staining, but readily labeled with anti-NKp46 antibodies applied to tissues sections ex vivo (Supplemental Figure 2C). Thus, both microscopy (Figure 1A) and intravascular staining (Figure 1, E and F) revealed few
WP-localized NK cells and a predominance of RP-localized NK cells in the absence of virus (Figure $1 \mathrm{~F}$ and Supplemental Figure 1). Following LCMV infection, the fraction (Figure 1F) and number (Figure 1E) of splenic iv-NKp46 $6^{\text {neg }} \mathrm{NK}$ cells increased over time, peaking at day 3 and returning to near baseline by day 6 of infection. Notably, differences in iv-NKp46 staining could not be explained by differences in ex vivo measurement of NKp46 expression levels (Supplemental Figure 2A). In total, these results show that NK cells transiently relocate to the WP during the first 3 days of LCMV infection, a window of time concomitant with perforindependent NK cell killing of activated T cells (4).

The intravascular staining method permitted comparison of the phenotype of NK cells in the WP (iv-NKp46 $6^{\text {neg }}$ ) and RP (iv-NKp46 ${ }^{+}$) after infection. Each subset exhibited similar expression levels of activating (Ly49H, DNAM-1, NKG2D) and inhibitory (CD94, NKG2A, KLRG1) NK cell receptors, although NKG2D 
A

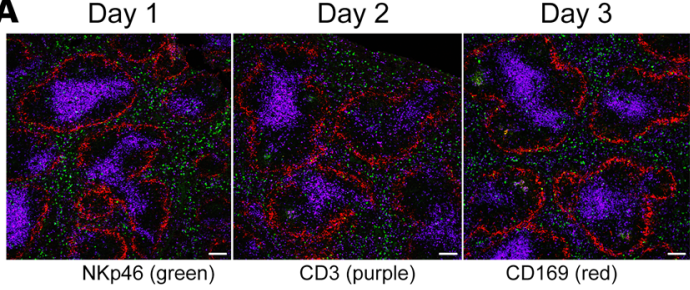

Day $3-\mathrm{Ad} 5$

\section{D}
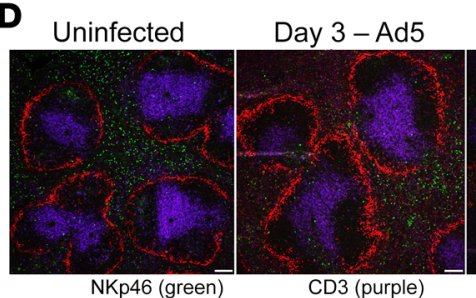

CD3 (purple)

CD169 (red)

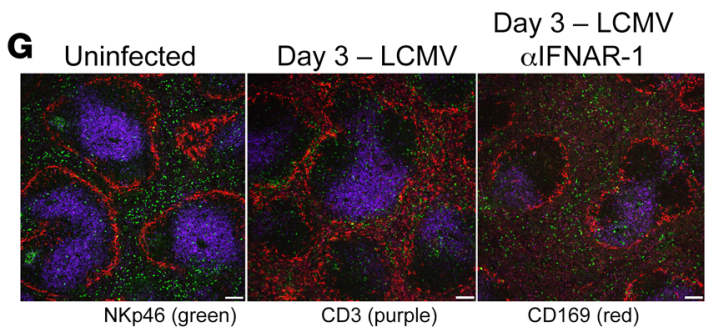

B

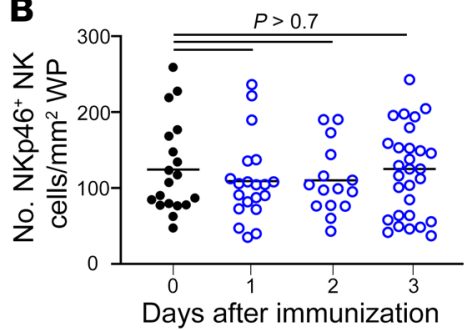

E

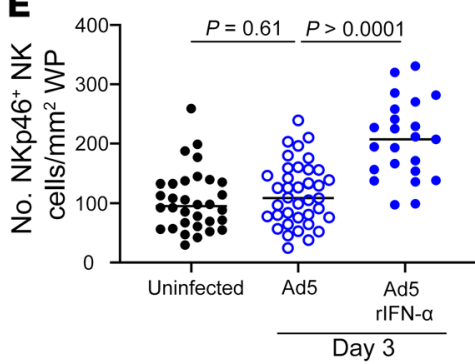

H

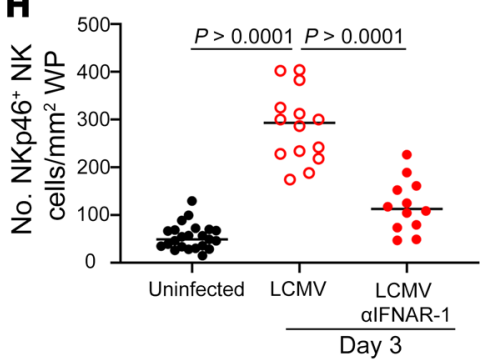

C
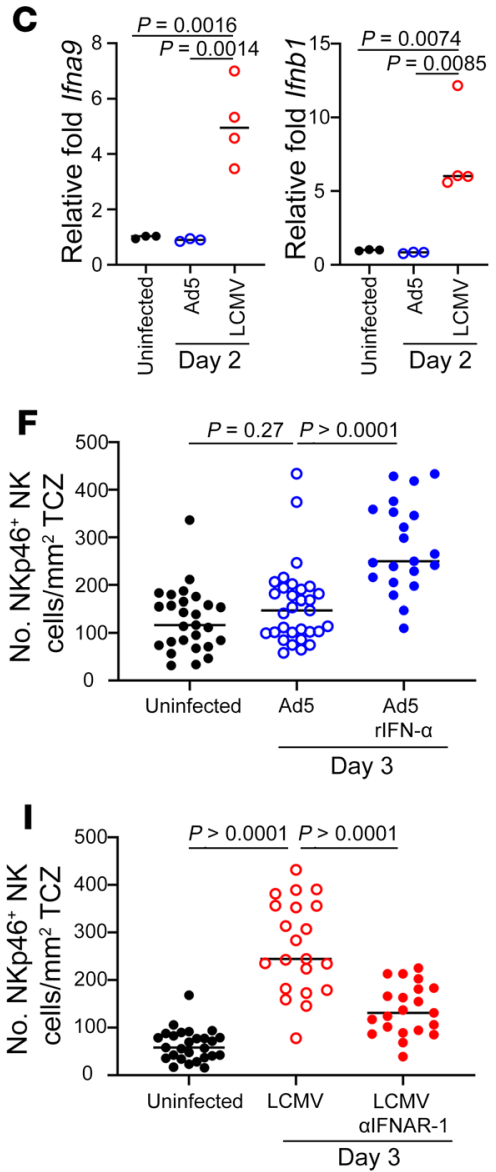

Figure 2. IFN-Is are necessary and sufficient to drive NK cell WP localization. C57BL/6 mice were inoculated with (A and B) Ad5-GP, (C-F) Ad5-LacZ, or (C, G-I) LCMV. One group (D-F) of Ad5-LacZ-inoculated C57BL/6 mice was treated with $8 \mu \mathrm{g} / \mathrm{d}$ recombinant IFN- $\alpha$, while another group (G-I) of LCMV-infected C57BL/6 mice was treated with $400 \mu \mathrm{g} / \mathrm{d}$ anti-IFNAR-1 antibody. At indicated time points, spleens were imaged (A, D, and G) to determine localization of $\mathrm{NKp} \mathrm{6}^{+} \mathrm{NK}$ cells (green) relative to $\mathrm{CD} 3^{+} \mathrm{T}$ cells (purple) and CD169+ macrophages (red), with enumeration of NK cells in (B, E, and H) CD169-delineated WP or ( $F$ and I) CD3+ T cell zones ( $n=3-4$ mice, 5-12 follicles/mouse). (C) Relative expression of Ifna9 and Ifnb1 2 days following LCMV infection or Ad5-LacZ inoculation compared with that of uninfected mice. Data are representative of 2 independent experiments, with statistically significant differences determined by 1-way ANOVA. Scale bars: $100 \mu \mathrm{m}$.

expression was slightly reduced on WP NK cells (Supplemental Figure 3, A-F). Expression of CXCR3 (Supplemental Figure 3G) as well as the distribution of immature $\left(\mathrm{CD} 11 \mathrm{~b}^{\text {neg }} \mathrm{CD} 27^{+}\right)$, transitional $\left(\mathrm{CD} 11 \mathrm{~b}^{+} \mathrm{CD} 27^{+}\right)$, and mature $\left(\mathrm{CD} 11 \mathrm{~b}^{+} \mathrm{CD} 27^{\text {neg }}\right) \mathrm{NK}$ cell subsets (Supplemental Figure 3H) were also similar between the RP and WP. However, WP NK cells expressed higher levels of granzyme B and the IL-2 receptor $\alpha$ (CD25) than RP NK cells (Supplemental Figure 3, I and J). Thus, WP NK cells may be more cytolytically active and IL-2 responsive than their counterparts in the RP.

Type I IFNs are necessary and sufficient to drive NK cell localization in WP. In contrast to LCMV infection, vaccination with a replication incompetent adenovirus serotype 5 vector (Ad5) triggered robust $\mathrm{T}$ cell responses without any evidence of $\mathrm{T}$ cell suppression by NK cells (21). We hypothesized that the absence of NK cell immunoregulatory functions after Ad5 vaccination may be associated with weak or absent induction of NK cell localization within T cell zones. As such, we immunized mice with replication-incompetent Ad5 vectors harboring either the glycoprotein (GP) of LCMV (ref. 21 and Figure 2, A and B) or $\beta$-galactosidase (Ad5-LacZ; Supplemental Figure 4 and Figure 2, C-F) and assessed NK cell local- ization. There was no measurable localization of NK cells within the WP at any time point measured after Ad5 immunization (Figure 2, A and B, and Supplemental Figure 4, A and B), supporting an association between NK cell migration and immune regulation.

One important distinction between LCMV infection and Ad5-vector immunization lies in the magnitude of type I IFN (IFN-I) responses, where LCMV is a potent inducer of IFN-I (22). Indeed, we measured an approximately 5 -fold increase in Ifna 9 and Infb1 expression during LCMV infection, but not Ad5 immunization (Figure 2C). Thus, we hypothesized that robust IFN-I expression during LCMV infection, but not after Ad5 immunization, promotes NK cell migration. Indeed, daily provision of recombinant IFN- $\alpha($ rIFN- $\alpha$ ) during Ad5 immunization resulted in increased NK cell localization within the WP (Figure 2, D and E) and $\mathrm{T}$ cell zones (Figure 2, D-F) of the spleen as well as enhanced NK cell accumulation in the draining lymph nodes (Supplemental Figure 5). In agreement with the importance of IFN-I for NK cell migration, addition of anti-IFN- $\alpha \beta$ receptor-blocking ( $\alpha$-IFNAR-1blocking) antibodies during LCMV infection impeded localization of NK cells in the WP (Figure 2, G and H) and T cell zones (Figure 
A
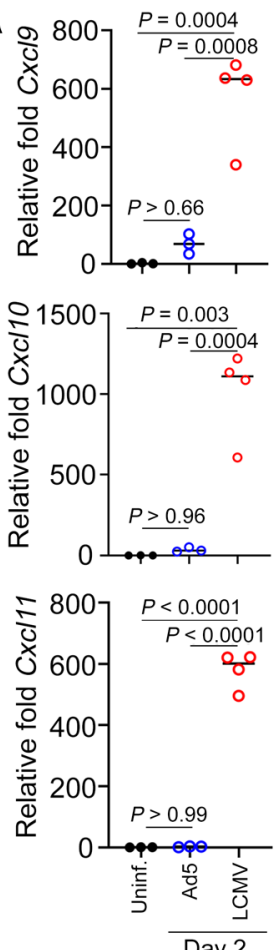

B

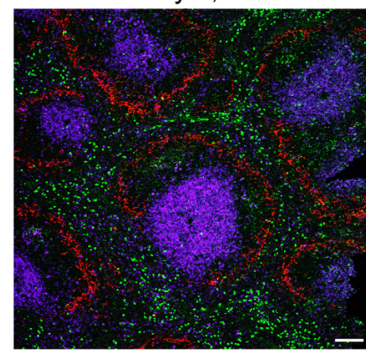

Day 3, CXCR3 Ko

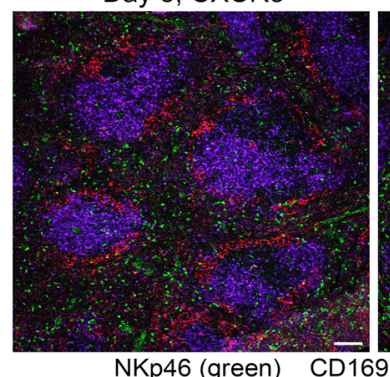

NKp46 (green) CD169 (red)
Day 3, WT

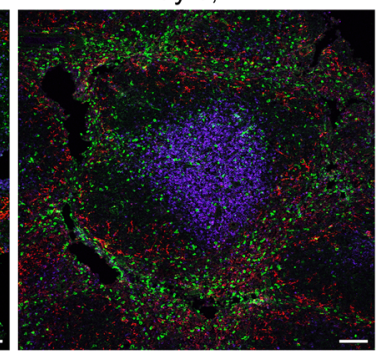

Day 3, CXCR3 ${ }^{\text {WT }}$

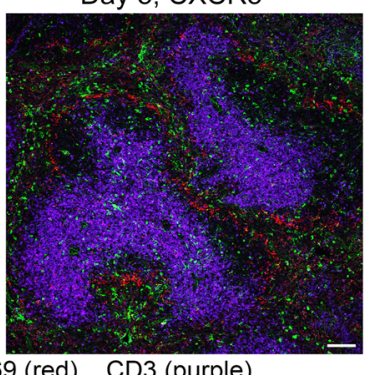

C

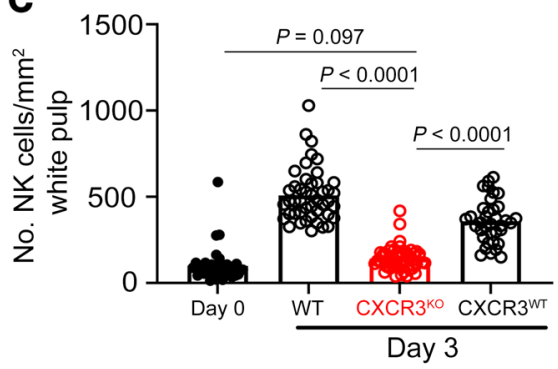

D

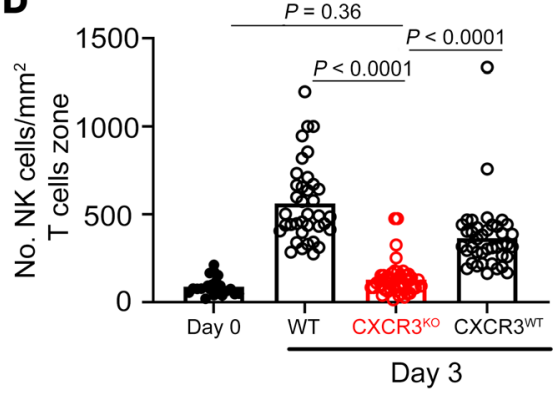

Figure 3. NK cells require CXCR3 for splenic T cell zone localization. (A) Relative expression of $C x C / 9, C x c / 10$, and $C x c / 11$ in the spleens of uninfected, Ad5LacZ inoculated, or LCMV-infected C57BL/6 mice ( $n=3-4 /$ group). (B-D) C57BL/6 mice (WT) and mixed CXCR3 ${ }^{\mathrm{WT}}$ or CXCR3 ${ }^{\mathrm{KO}}$ bone marrow chimeras ( $n=3$ ) were infected with LCMV. Prior to (day 0) or after (day 3) infection, (B) confocal microscopy was used to determine median number (5-12 follicles/mouse) of NKp46 NK cells (green) in (C) CD169+ macrophage-bordered (red) WP or in (D) CD3 ${ }^{+}$T cell zones (purple). Data are representative of 2 independent experiments, with statistically significant differences determined by 1-way ANOVA. Scale bars: $100 \mu \mathrm{m}$.

2, G-I) of the spleen. IFN-I blockade also reduced NK cell accumulation in draining lymph nodes after LCMV infection (Supplemental Figure 5). Thus, IFN-Is are important triggers of NK cell migration to $\mathrm{T}$ cell-rich sites in lymphoid tissues.

$N K$ cells require CXCR3 for splenic $T$ cell zone localization. Inflammatory recruitment of NK cells to lymph nodes during poxvirus infection or after dendritic cell immunization requires expression of the chemokine receptors CXCR3 on NK cells (17, 23, 24). Likewise, CXCR3 is implicated in NK cell localization to the WP after polyinosinic:polycytidylic acid (poly I:C) injection or MCMV infection $(14,15)$. Therefore, we hypothesized that IFN-I induction of CXCR3 ligands and CXCR3 expression on NK cells is vital for migration to $\mathrm{T}$ cell zones in the WP. The expression of the CXCR3 ligands Cxcl9, Cxcl10, and Cxcl11 was elevated following LCMV infection, but not Ad5 immunization (Figure 3A). IFNAR-1 blockade dampened LCMV-mediated induction of Cxcl10 and Cxcl11 (Supplemental Figure 6).

To address the role of CXCR3 in NK cell migration and suppressive function without undermining any role for CXCR3 in T cell activation, we generated mixed bone marrow chimeric (BMC) mice harboring CXCR3-sufficient $\mathrm{T}$ and $\mathrm{B}$ cells in conjunction with an innate compartment that was either CXCR3 sufficient or deficient. $\mathrm{Rag}^{K O} \mathrm{Cxcr} 3^{W T}$ or $\mathrm{Rag}^{K O} \mathrm{Cxcr} 3^{\mathrm{KO}}$ bone marrow cells (Ly5.2) were mixed at a 9:1 ratio with WT bone marrow cells (Ly5.1) prior to reconstitution of lethally irradiated mice. This protocol (25) ensures that RAG-dependent cells, including $\mathrm{T}$ and $\mathrm{B}$ cells, are ubiquitously CXCR3 sufficient while innate cells such as
NK cells are predominately derived from the Rag ${ }^{K O}$ precursors that are either $\mathrm{Cxcr} 3$ sufficient or deficient. We confirmed that CXCR3 was absent from NK cells in the CXCR ${ }^{\mathrm{KO}}$ chimeras (Supplemental Figure $7 \mathrm{~A}$ ). The resulting $\mathrm{CXCR}^{\mathrm{KO}}$ and $\mathrm{CXCR} 3^{\mathrm{WT}}$ chimeric mice were infected with LCMV prior to assessment of NK cell localization on day 3 of infection (Figure 3B). Similarly to what occurred with infected nonchimeric C57BL/6 mice (WT), a substantial fraction of NK cells colocalized within the WP (Figure 3C) and T cell zones (Figure 3D) of infected CXCR ${ }^{\mathrm{WT}} \mathrm{BMC}$ mice. In contrast, NK cell frequencies within the WP (Figure $3 C$ ) and $T$ cell zones (Figure 3D) were significantly reduced in CXCR3 ${ }^{\mathrm{KO}}$ BMCs. Thus, localization of NK cells within T cell-rich regions of the WP during LCMV infection depends on NK cell expression of CXCR3.

CXCR3 is required for NK cell suppression of antiviral $T$ cells. Transient localization of NK cells in the WP during LCMV infection (Figure 1) coincides with the window of time during which NK cells kill activated T cells (4). Since CXCR3 is necessary for NK cell localization within T cell-rich WP during LCMV infection (Figure 3), we hypothesized that mice lacking CXCR3 on NK cells would display an enhanced virus-specific $\mathrm{T}$ cell response similar to that of mice depleted of NK cells. Anti-NK1.1 antibody treatment 1 day before infection effectively depleted NK cells from both sets of mixed BMC mice (Supplemental Figure 7B). At day 7 of LCMV infection, antiviral $\mathrm{T}$ cell responses were assessed by intracellular cytokine staining after in vitro restimulation with viral peptide. The proportion (Figure 4A) and number (Figure 4B) of IFN- $\gamma^{+}$ $\mathrm{TNF}^{+} \mathrm{LCMV} \mathrm{GP}_{64-80}$-specific CD $4^{+} \mathrm{T}$ cells was elevated to a similar 
A

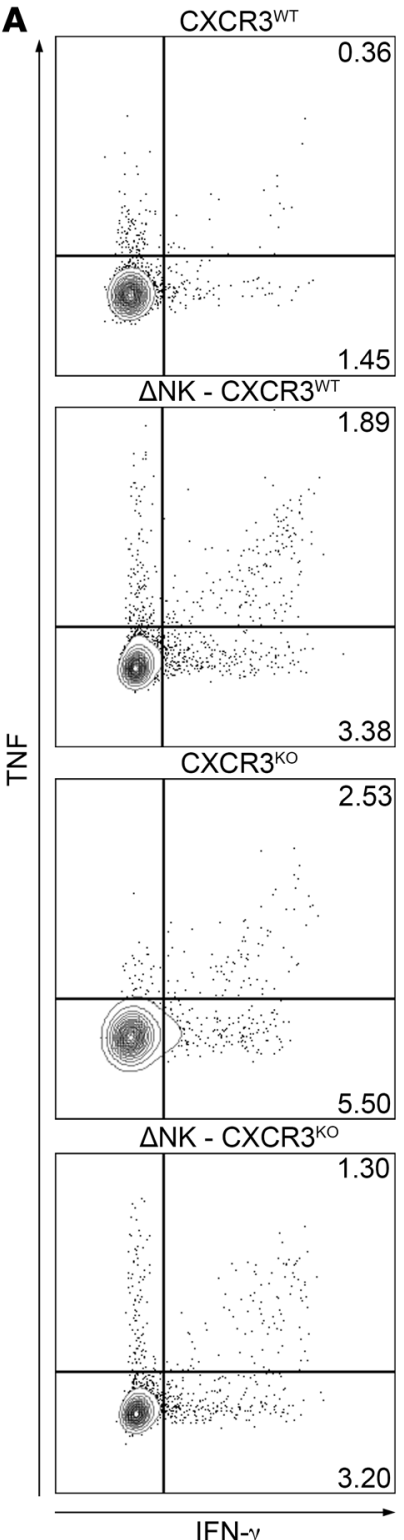

B

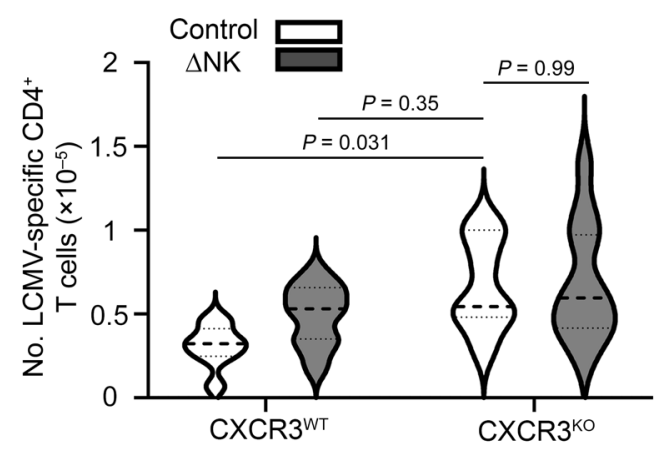

C

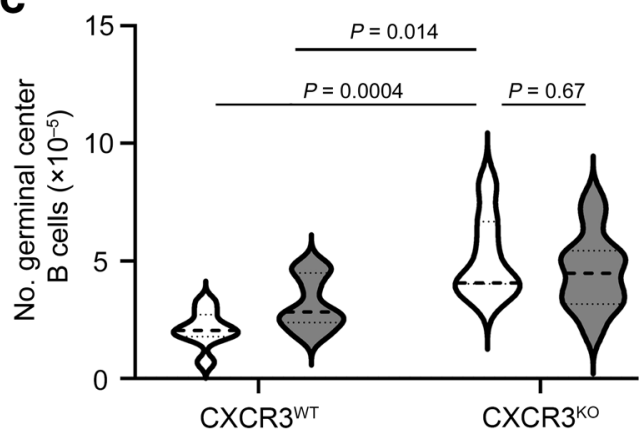

D

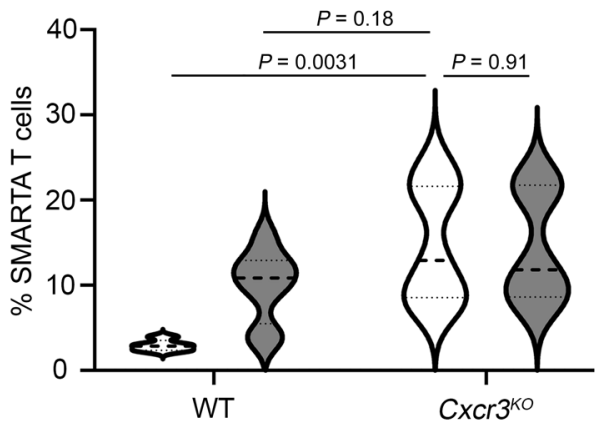

Figure 4. CXCR3 is required for NK cell suppression of antiviral T cells. (A-C) Mixed CXCR3 ${ }^{\mathrm{WT}}$ and $\mathrm{CXCR}^{\mathrm{KO}}$ bone marrow chimeric mice ( $n=7-12$ mice) group) were depleted of NK cells via anti-NK1.1 ( $\triangle \mathrm{NK})$ or control antibody 1 day prior to LCMV infection. (B) Numbers of IFN- $\gamma^{+} \mathrm{TNF}^{+}$-expressing LCMV GP ${ }_{64-80^{-}}$ specific $C D 4^{+} T$ cells were measured on day 7 by intracellular cytokine staining and flow cytometry. (C) Numbers of Fas ${ }^{+} \mathrm{CL} 7^{+}$germinal center $\mathrm{B}$ cells were measured. (D) $C 57 \mathrm{BL} / 6$ and $\mathrm{CxCr}^{\mathrm{KO}}(n=3-4$ mice $/$ group) mice were depleted or not of NK cells ( $\triangle N K$ ) prior to intravenous infusion of $5 \times 10^{5} \mathrm{LCMV}$-specific Ly5.1+ transgenic SMARTA CD4+ $T$ cells and infected 1 day later with LCMV. At day 6, the proportions of donor TCR-V $\alpha 2^{+}$Ly5.1+CD4+ SMARTA T cells were quantified in spleens of recipient mice. Statistical analyses were performed using 1-way ANOVA. Data are pooled from 2 independent experiments.

after infection and in the resulting suppression of antiviral $\mathrm{T}$ cells by NK cells. We demonstrate that IFN-I-dependent induction of CXCR3 ligand expression is a vital mechanism determining NK cell localization in T cell zones. Adenoviral vectors that do not robustly induce IFN-I also fail to elicit NK cell follicular migration and associated suppression of $\mathrm{T}$ cells. Therefore, CXCR3 directs crucial redistribution of NK cells during inflammation that brings these cells into close proximity of activated $\mathrm{T}$ cells to permit perforin-dependent regulation (Supplemental Figure 8). Translational targeting of this mechanism represents an innovative means of potentially enhancing vaccine efficacy and boosting antibody generation.

The transient nature of WP localization of NK cells represents a mechanistic explanation for the narrow temporal window of immunoregulatory function of NK cells. Measurable killing of activated $\mathrm{T}$ cells by NK cells is limited to the first 3 days of acute LCMV infection $(3,4)$, over-

extent in CXCR3 ${ }^{\mathrm{KO}}$ chimeras and in NK cell-depleted CXCR3 ${ }^{\mathrm{WT}}$ chimeras, with no apparent additive effect of NK cell depletion in CXCR3 ${ }^{\mathrm{KO}}$ chimeras. The numbers of splenic $\mathrm{Fas}^{+} \mathrm{GL}^{+}$germinal center B cells were similarly increased by CXCR3 deficiency or NK cell depletion (Figure 4C).

As an alternative test of our hypothesis, CXCR3-sufficient LCMV-specific TCR transgenic SMARTA CD $4^{+}$T cells (Ly5.1) were seeded in either CXCR3-sufficient C57BL/6 mice or germline Cxcr ${ }^{K O}$ mice (Ly5.2) prior to NK cell depletion and LCMV inoculation. On day 6 of infection, the frequencies of donor SMARTA cells expressing IFN- $\gamma$ and TNF were increased in $\mathrm{C} x \mathrm{cr} 3^{\mathrm{KO}}$ and NK cell-depleted recipient mice relative to those in control animals (Figure 4D). There was no additional effect of NK cell depletion in $\mathrm{Cxcr}^{\mathrm{KO}}$ hosts. Thus, CXCR3 expression is important for NK cell suppression of antiviral $\mathrm{T}$ cells.

These data reveal a crucial spatiotemporal mechanism of NK cell regulation of antiviral $\mathrm{T}$ cells. We identify CXCR3 as essential for both transient localization of NK cells within $\mathrm{T}$ cell zones lapping with the peak localization of NK cells in the WP reported here. Rapid egress or loss of NK cells from T cell zones after day 3 likely limits further immunoregulatory killing. The degree to which different pathogens or vaccines trigger CXCR3-dependent migration of NK cells is linked to the ability to induce IFN-I responses. Some adenoviral vectors (e.g., Ad28 and Ad35) do trigger IFN-I release (26), but these responses are several orders of magnitude lower than those seen after LCMV infection (22) and rapidly wane within hours of inoculation.

In addition to LCMV, relocalization of NK cells to $\mathrm{T}$ cell zones (14-16) and NK cell suppression of T cells are also triggered during infections with MCMV or after injection of TLR ligands (4). Induction of IFN and ligands for CXCR3 (27) are shared features of these contexts. This explains why viruses such as vaccinia virus and Ad5 vectors that weakly induce IFN responses $(26,28)$ also poorly stimulate NK cell inhibition of T cells $(21,29)$. Of note, transient blockade of IFN-I early during LCMV infection can enhance antiviral $\mathrm{T}$ and $\mathrm{B}$ cell responses (30). Ablation of the IFNAR on NK 
cells exerts a similar effect (31). In MCMV and SIV/HIV infections, in which NK cells exhibit potent antiviral function, this migration of NK cells to $\mathrm{T}$ cell zones serves to protect these tissues from virus $(15,19)$. However, conservation of this migration during immunization or during infection with viruses that are refractory to NK cell antiviral functions (e.g., LCMV) is more consequential for immune regulation than for immune defense.

Our finding of CXCR3-mediated, WP-localizing NK cells in suppression of $\mathrm{T}$ cells opens avenues of research that will enhance our understanding of the biology of this process as well as provide translational targets to circumvent this activity of NK cells. Immunosuppressive function of NK cells is linked to reduced $\mathrm{T}$ cell memory (3), neutralizing antibody titers $(3,8)$, antibody affinity maturation (6), and vaccine efficacy (10). Our research highlights the potential for interventions blocking CXCR3 or its ligands in NK cell immunosuppression to enhance vaccine efficacy. Further characterization of WP-localized NK cells is likely to reveal key mediators involved in T cell suppression. Indeed, WP- and RPlocalized NK cells exhibit markedly different transcriptomes (our unpublished observations). This represents a promising pipeline for identification of translational targets to enhance vaccineelicited immune responses by circumventing NK cell activity.

\section{Methods}

Statistics. Experimental results are consistently presented as the median with individual data point spread. Statistical differences between control and experimental groups were calculated using 1-way ANOVA with either the Holm-Šídák multiple-comparison test or a Dunnett's multiple-comparison test. When a confidence interval was not desired, the Holm-Šídák multiple-comparison method was used to generate higher statistical power. A $P$ value of less than 0.05 was considered significant. Graphing and statistical analysis were rou- tinely performed using GraphPad Prism. Researchers were blinded to groupings and treatment during experimental measurements.

Additional details are presented in the Supplemental Methods.

Study approval. All animal studies were approved by the Institutional Animal Care and Use Committee and the Institutional Biosafety Committee of Cincinnati Children's Hospital Medical Center.

\section{Author contributions}

AA, MTM, and SNW conceived and designed the study. AA, LMC, HAF, HC, MTM, JAT, NL, SR, HS, and DK performed experiments and acquired data. AA, LMC, HAF, HC, MTM, JAT, NL, SR, SNW, $\mathrm{EB}$, and $\mathrm{DHB}$ provided reagents. AA and $\mathrm{SNW}$ drafted the manuscript. AA, LMC, HAF, HC, MTM, JAT, NL, SR, HS, DK, EB, DHB, and SNW provided critical editing of the manuscript.

\section{Acknowledgments}

We thank M. Kofron and the Comprehensive Mouse and Cancer Core for support, J. Molkentin for Ad-LacZ, S. Crotty for SMARTA mice, Shane D'Souza for crafting the summary model, and L. Kottyan, I. Lewkowich, D. Hildeman, R. Herro, and M. Borchers for critical reading of the manuscript. Funding for this work was provided by NIH grants DA038017, AI148080, and AR073228 (to SNW); T32GM063483 (to AA, NL, and HAF); and T32AI118697 (to AA). Support was also provided by the Cincinnati Children's Research Foundation (to SNW), the L.B. Research and Education Foundation (to NL), and the Albert J. Ryan Foundation (to AA). The Flow Cytometry Core was supported by NIH grants AR070549 and DK078392.

Address correspondence to: Stephen Waggoner, 3333 Burnet Avenue, Cincinnati, Ohio 45229, USA. Phone: 513.803.4607; Email: Stephen.Waggoner@cchmc.org.
1. Mace EM, et al. Genetic causes of human NK cell deficiency and their effect on NK cell subsets. Front Immunol. 2016;7:545.

2. Waggoner SN, et al. Roles of natural killer cells in antiviral immunity. Curr Opin Virol. 2016;16:15-23.

3. Rydyznski C, et al. Generation of cellular immune memory and B-cell immunity is impaired by natural killer cells. Nat Commun. 2015;6:6375.

4. Waggoner SN, et al. Natural killer cells act as rheostats modulating antiviral T cells. Nature. 2011;481(7381):394-398.

5. Lang PA, et al. Natural killer cell activation enhances immune pathology and promotes chronic infection by limiting CD8+ T-cell immunity. Proc Natl Acad Sci U S A. 2012;109(4):1210-1215.

6. Rydyznski CE, et al. Affinity maturation is impaired by natural killer cell suppression of germinal centers. Cell Rep. 2018;24(13):3367-3373.

7. Abruzzo LV, et al. Homeostasis of the antibody response: immunoregulation by NK cells. Science. 1983;222(4624):581-585.

8. Bradley T, et al. RAB11FIP5 expression and altered natural killer cell function are associated with induction of HIV broadly neutralizing antibody responses. Cell. 2018;175(2):387-399.

9. Peppa D, et al. Up-regulation of a death receptor renders antiviral $\mathrm{T}$ cells suscepti- ble to NK cell-mediated deletion. J Exp Med. 2013;210(1):99-114.

10. Muyanja E, et al. Immune activation alters cellular and humoral responses to yellow fever 17D vaccine. J Clin Invest. 2014;124(7):3147-3158.

11. De Mot L, et al. Transcriptional profiles of adjuvanted hepatitis B vaccines display variable interindividual homogeneity but a shared core signature. Sci Transl Med.2020;12(569):eaay8618.

12. Soderquest $\mathrm{K}$, et al. Cutting edge: $\mathrm{CD} 8+\mathrm{T}$ cell priming in the absence of NK cells leads to enhanced memory responses. JImmunol. 2011;186(6):3304-3308.

13. Voskoboinik I, et al. Perforin and granzymes: function, dysfunction and human pathology. Nat Rev Immunol. 2015;15(6):388-400.

14. Gregoire C, et al. Intrasplenic trafficking of natural killer cells is redirected by chemokines upon inflammation. Eur J Immunol. 2008;38(8):2076-2084.

15. Bekiaris V, et al. Ly49H+ NK cells migrate to and protect splenic white pulp stroma from murine cytomegalovirus infection. J Immunol. 2008;180(10):6768-6776.

16. Salazar-Mather TP, et al. NK cell trafficking and cytokine expression in splenic compartments after IFN induction and viral infection. JImmunol.1996;157(7):3054-3064.
17. Martin-Fontecha A, et al. Induced recruitment of NK cells to lymph nodes provides IFN-gamma for $\mathrm{T}(\mathrm{H}) 1$ priming. Nat Immunol. 2004;5(12):1260-1265.

18. Ferlazzo G, et al. The abundant NK cells in human secondary lymphoid tissues require activation to express killer cell Ig-like receptors and become cytolytic. JImmunol. 2004;172(3):1455-1462.

19. Huot N, et al. Natural killer cells migrate into and control simian immunodeficiency virus replication in lymph node follicles in African green monkeys. Nat Med. 2017;23(11):1277-1286.

20. Anderson KG, et al. Intravascular staining for discrimination of vascular and tissue leukocytes. Nat Protoc. 2014;9(1):209-222.

21. Blass E, et al. Adenovirus vector vaccination impacts NK cell rheostat function following lymphocytic choriomeningitis virus infection. J Virol. 2018;92(11):e02103-17.

22. Biron CA. Cytokines in the generation of immune responses to, and resolution of, virus infection. Curr Opin Immunol. 1994;6(4):530-538.

23. Wong E, et al. Migratory dendritic cells, group 1 innate lymphoid cells, and inflammatory monocytes collaborate to recruit NK Cells to the virus-infected lymph node. Cell Rep. 2018;24(1):142-154.

24. Pak-Wittel MA, et al. Interferon- $\gamma$ mediates 
chemokine-dependent recruitment of natural killer cells during viral infection. Proc Natl Acad Sci U S A. 2013;110(1):E50-E59.

25. Kurtulus S, et al. Bim controls IL-15 availability and limits engagement of multiple $\mathrm{BH} 3$-only proteins. Cell Death Differ. 2015;22(1):174-184.

26. Johnson MJ, et al. Type I IFN induced by adenovirus serotypes 28 and 35 has multiple effects on $\mathrm{T}$ cell immunogenicity. J Immunol.
2012;188(12):6109-6118.

27. Kelly-Scumpia KM, et al. Type I interferon signaling in hematopoietic cells is required for survival in mouse polymicrobial sepsis by regulating CXCL10. JExp Med. 2010;207(2):319-326.

28. Perdiguero B, et al. The interferon system and vaccinia virus evasion mechanisms. J Interferon Cytokine Res. 2009;29(9):581-598.

29. Hatfield SD, et al. Weak vaccinia virus-induced
NK cell regulation of CD4 T cells is associated with reduced NK cell differentiation and cytolytic activity. Virology. 2018;519:131-144.

30. Palacio N, et al. Early type I IFN blockade improves the efficacy of viral vaccines. J Exp Med. 2020;217(12):e20191220.

31. Huang Z, et al. IFNAR1 signaling in NK cells promotes persistent virus infection. Sci $A d v$. 2021;7(13):eabb8087. 